\title{
Editorial
}

\section{Haematuria: Do we always know the answer?}

Normal urine should contain less than three red blood cells per HPF. Macroscopic (gross) haematuria means the patient has seen blood in urine, whereas in microscopic or dipstick haematuria, blood is identified by urine microscopy or by dipstick testing, either in association with other urological symptoms (symptomatic microscopic haematuria) or during a routine medical examination (asymptomatic microscopic haematuria-AMH).

The sensitivity of urinary dipsticks in identifying haematuria is over $90 \%$ whereas the specificity of the dipstick for haematuria compared with microscopy is somewhat lower. Haematuria, haemoglobinuria, and myoglobinuria will all result in positive dipstick for blood. Therefore, the dipstick results should be confirmed with a microscopic examination of the centrifuged urinary sediment.

Haematuria may reflect either significant nephrologic or urologic disease. Haematuria of nephrologic origin is frequently associated with casts in the urine and almost always associated with significant proteinuria. Even significant haematuria of urologic origin will not elevate the protein concentration in the urine up to the 100 to $300 \mathrm{mg} / \mathrm{dl}$ and proteinuria of this magnitude almost always indicates glomerular or tubulointerstitial renal disease. Morphologic evaluation of erythrocytes in the centrifuged urinary sediment also helps localize their site of origin. Erythrocytes arising from glomerular disease are typically dysmorphic. Nonglomerular haematuria or essential haematuria includes primarily urologic (surgical) diseases. Common causes are urologic tumors, stones, and UTIs.

Haematuria without other symptoms (painless) must be regarded as a symptom of tumor of the bladder or kidney until proved otherwise. It is usually intermittent; bleeding may not recur for months. Complacency because the bleeding stops spontaneously must be condemned. Less common causes of silent haematuria are staghorn calculus, polycystic kidneys, benign prostatic hyperplasia, solitary renal cyst, sickle cell disease, and hydronephrosis. Painless bleeding is common with acute glomerulonephritis.

Joggers and people who engage in participatory sports frequently develop transient proteinuria and gross or microscopic haematuria.

Investigations usually involve urine culture and cytology, cystoscopy, and upper tract imaging by renal ultrasound with or without intravenous urogram (IVU) in most patients. Elderly patients with gross haematuria may need CT Urogram (contrast CT scan of KUB region) for upper tract evaluation.

Studies have shown that the incidence of malignancy ranges from 2 to $22 \%$ for elderly patients with AMH and the chances are very low for those aged below 50 years. The only treatable urological conditions in this younger group are UTI and stone disease, both of which are symptomatic and need investigations on their own merits. Therefore, patients aged below 50 years and AMH could be managed with little or no urological investigation and should be referred for a nephrological opinion, as renal parenchymal disease is more likely. In contrast, gross haematuria is a red flag symptom requiring urgent urology referral and evaluation. The reported incidence of urological cancer in patients presenting with gross haematuria is between $19 \%$ and $25 \%$, and these are commonly bladder and renal tumors. In addition, the incidence of benign surgical disease associated with gross haematuria is $21.4 \%$. However, about $80 \%$ of patients with gross haematuria who receive no diagnosis during the initial standard investigations will not have recurrent haematuria. For remaining $20 \%$ of patients who have recurrent gross haematuria, repeat investigations are mandatory because about half $(9.8 \%)$ of them might be diagnosed as having urological malignancy 
The fact that the presence of RBCs in urine is normal; explains why a substantial proportion of patients with microscopic haematuria, and even macroscopic haematuria will have normal haematuria investigations (i.e. no abnormality is found).

The upper limit of normal for $\mathrm{RBC}$ excretion is 1 million per $24 \mathrm{~h}$; that means about $1000 \mathrm{RBC} / \mathrm{ml}$ of urine, range could be as from 5000 to $8000 \mathrm{RBC} / \mathrm{ml}$. Because each HPF views between $1 / 20,000$ and $1 / 50,000 \mathrm{mls}$ of urine, each RBC seen per HPF signifies more than $20,000 / \mathrm{ml}$. Therefore, $3 \mathrm{RBC} / \mathrm{HPF}$ reflects counts of about $60,000 / \mathrm{ml}$ that is $7-12$ times higher than normal excretion. Therefore, transient microscopic haematuria may occur following rigorous exercise, sexual intercourse, or from menstrual contamination and in dehydrated person.
We can conclude that, despite a standard set of investigations a significant proportion of patients will receive no etiological diagnosis but persistence of gross haematuria always warrants further investigations. The incidence of underlying malignancy in patients with AMH and aged $>50$ years makes their investigation worthwhile. It is more difficult to justify investigating younger patients with AMH.

\section{Dr Md Mohsin Uddin}

Joint Editor

Associate Professor of Urology, KYAMCH 